\title{
Interference effects in tone memory
}

\author{
RICHARD K. OLSON \\ University of Colorado, Boulder, Colorado 80309 \\ and \\ VICKI HANSON \\ University of Oregon, Eugene, Oregon 97403
}

\begin{abstract}
Memory for a standard tone in comparison to a subsequent test tone was examined in three experiments with three intervening tones between the standard and test tones. In each trial, the intervening tones were presented from one of seven frequency range and distance from the standard tone conditions. Experiment 1 tone patterns were played at four different presentation rates, and the subjects judged whether the test was higher or lower than the standard. Memory interference effects caused by the different intervening tone conditions could be accounted for by a directional shift in the standard tone memory toward the intervening tones and by a general decrease in the standard tone memory strength with more distant intervening tones. Interference effects were smaller for the rapid presentation rates because the intervening tones formed separate "perceptual streams." Two additional experiments presented the tone patterns in a task requiring the subjects to match a continuously variable tone to their memory of the standard (Experiment 2) and a task requiring them to judge whether the standard and test tones were the "same" or "different" (Experiment 3). These experiments showed large differences in interference effects as a function of the required judgment and the subjects' musical experience.
\end{abstract}

Memory for the pitch of a tone is disrupted when it is followed by tones of a different pitch. Deutsch and her colleagues have explored this effect in a series of studies which typically involved the sequential presentation of a standard tone, several intervening tones, and a test tone that the subject judged as "same as" or "different from" the standard (see Deutsch, 1975, for a review). In most of these studies, the effects of intervening tones were closely examined only for relatively small frequency separations from the standard and test tones (e.g., Deutsch \& Feroe, 1975). This line of research has provided some important insights into the "microstructure" of intervening tone effects on pitch memory, but relatively little is known about the effects of larger intervening tone separations from the standard tone.

The present series of studies tested the effects of intervening tones which ranged from 1 to 20 semitones above or below the standard tone. (One semitone is equal to a frequency difference ratio of about $1: 1.06$.) The experiments were designed to explore both the

This research was supported by the Council on Research and Creative Work at the University of Colorado and the Institute for the Study of Intellectual Behavior. Janet Fletcher and Darci Glassman performed pilot studies for the first experiment, and Ronald Leatherman and John Durrett assisted in the construction of stimulus materials. Larry Greenblatt, Anne Frey, and Martha Timyan performed a pilot study for the second experiment. Jan Keenan assisted with the data analysis for the third experiment and commented on an earlier draft of this paper. Portions of the paper were presented at the Denver meetings of the Psychonomic Socicty, November 1975. general strength of the standard tone memory, and, more specifically, the direction of memory distortion as a function of different intervening tone conditions. We initially considered the following three hypotheses and models to predict the effects of intervening tones as a function of their frequency separation from the standard and test tones.

1 . Tone memory would be maximally disrupted by intervening tones closest to the standard and test. The adjacency of encoding locations on the basilar membrane and in the central nervous system would result in maximum interaction and distortion of memory traces for tones adjacent on the frequency dimension.

2 . Tone memory would be minimally disrupted by intervening tones closest to the standard and test. Memory for tone patterns involves a relational encoding system (Attneave \& Olson, 1971; Deutsch, 1969), and the accuracy of relational encoding decreases with larger frequency differences between tones for most subjects (Attneave \& Olson, 1971).

3. A minimal interference effect might also occur for intervening tones closest to the standard and test tones because of adaptation level effects. According to this model, the memory representation of the standard would become a weighted average of the frequencies of the standard and intervening tones, and more distant intervening tones would result in a larger shift in the standard memory.

Note that the relational encoding and adaptation level models both predict minimal interference for intervening tones closest to the standard and test. However, the 
models differ in the type of memory distortion caused by intervening tones more distant from the standard and test. The relational encoding model simply predicts an increase in variance for the standard memory. The adaptation level model predicts a directional shift in the memory for the standard toward the frequency of the intervening tones.

Three experiments were performed to test the above models of intervening tone effects. In Experiment 1, each of the 56 trials consisted of five sequentially presented tones: a standard tone, three intervening tones selected from one of seven frequency range conditions, and a final test tone which the subject judged as higher or lower than the standard. The trial series was presented to four groups of subjects under four intertone temporal interval conditions: $300 \mathrm{msec}, 1 \mathrm{sec}, 3 \mathrm{sec}$, and $5 \mathrm{sec}$.

The results of Experiment 1 seemed to support the adaptation level model, but there was a suggestion that some of the evidence for a shift in the standard tone memory was artifactually generated by the response measure. Two additional experiments tested for shifts in the standard tone memory with different response measures. Experiment 2 presented the $1-\mathrm{sec}$ and $3-\mathrm{sec}$ intertone trial series without the final test tone, and required the subject to set a variable oscillator to his memory of the standard tone. Experiment 3 presented the same 3-sec intertone interval series as in Experiment 1, but the subjects were instructed to respond whether the standard and test tones sounded the "same" or "different."

\section{EXPERIMENT 1}

\section{Method}

Subjects. One hundred and four students from introductory psychology courses at the University of Colorado participated in the experiment to fulfill a research participation requirement.

Stimulus materials and conditions. All tones were taken from a musical scale $(A=440 \mathrm{~Hz})$. The basic pattern in each trial consisted of one of four standard tones, three intervening tones chosen from one of the seven frequency range conditions described below, and a test tone that was two semitones higher or lower than the standard. The standard tone frequency choices (in cycles/second) were $659,698,739$, and 783. The test tone frequency choices were $587,622,659,698,739,783,830$, and 880 . The seven frequency range intervening tone conditions are presented in Table 1. Note that the "low" and "high" conditions included tones which were separated from the standard tones by at least four semitones and not more than nine semitones. The

Table 1

The Seven Intervening Tone Frequency Conditions

\begin{tabular}{ll}
\hline \multicolumn{1}{c}{ Condition } & Intervening Tones (Hertz) \\
\hline Lowest & $246,261,277$ \\
Low & $466,493,523$ \\
Middle & $659,698,739,783$ \\
High & $987,1046,1108$ \\
Highest & $1864,1975,2093$ \\
Middle-Lowest & 2 middle tones, 1 lowest \\
Middle-Highest & 2 middle tones, 1 highest \\
\hline
\end{tabular}

Table 2

The Four Temporal Intervals

\begin{tabular}{cccc}
$\begin{array}{c}\text { Temporal } \\
\text { Interval* }\end{array}$ & $\begin{array}{c}\text { Duration of } \\
\text { Tones }\end{array}$ & $\begin{array}{c}\text { Temporal } \\
\text { Interval** }\end{array}$ & $\begin{array}{c}\text { Intertrial } \\
\text { Interval }\end{array}$ \\
\hline $5 \mathrm{sec}$ & $1 \mathrm{sec}$ & $5 \mathrm{sec}$ & $10 \mathrm{sec}$ \\
$3 \mathrm{sec}$ & $1 \mathrm{sec}$ & $3 \mathrm{sec}$ & $18 \mathrm{sec}$ \\
$1 \mathrm{sec}$ & $1 \mathrm{sec}$ & $1 \mathrm{sec}$ & $26 \mathrm{sec}$ \\
$300 \mathrm{msec}$ & $200 \mathrm{msec}$ & $2 \mathrm{sec}$ & $31.1 \mathrm{sec}$ \\
\hline
\end{tabular}

*Between standard and intervening tones

**Between last intervening and test tones

"highest" and "lowest" conditions were at least 15 semitones and not more than 20 semitones from the standard. The "middle" condition consisted of three of the four possible standard tones, excluding the particular standard for that trial. Intervening tones were randomly selected with the constraint that no tone was repeated within a trial. Finally, the "middlelowest" and "middle-highest" intervening tones were selected from the "middle" range in the first two positions, and one tone was selected from the "lowest" or "highest" condition for the third position.

The eight possible combinations of standard and comparison tones (separated by two semitones higher or lower) were presented with each of the seven intervening tone conditions, yielding a total of 56 trials. The trials were ordered in the following manner. Each standard was presented once in random order in each block of four trials, with the constraint that the standard presented at the end of a block would not be the same as the standard beginning the next block. Intervening tone conditions were randomly assigned to positions in the stimulus series, with the constraint that trials from the same intervening condition were not repeated consecutively, and each intervening condition was presented four times in the first half of the series (28 trials) and four times in the second half. One trial order was prepared by the above method and a second trial order was generated by reversing the first order.

The two series of tones described above were each recorded under the four different temporal interval conditions presented in Table 2. Note that in the 1-, 3-, and 5-sec interval conditions, the tone durations were always $1 \mathrm{sec}$. Although the intertone intervals varied with the different temporal conditions, the time between the beginning of one trial and the beginning of the next trial was a constant $35 \mathrm{sec}$, yielding a trial series duration of $32 \mathrm{~min} 40 \mathrm{sec}$ for each of the eight tapes (4 temporal conditions by 2 orders). The beginning of each trial was cued 2 sec prior to the standard tone by a $50-\mathrm{msec} 1,000-\mathrm{Hz}$ tone.

Apparatus for generation and presentation of stimuli. Pure tone stimuli were generated by a Wavetec oscillator which was driven by a Xerox Sigma 3 computer. The output was recorded and played to subjects on a SONY Model TC-570, with a loudspeaker that accompanied the tape recorder.

Procedure and instructions to subjects. Eight groups of 13 subjects each heard one of the eight tapes. They were seated about $10 \mathrm{ft}$ from the loudspeaker and were instructed to listen to the standard tone, compare its pitch with the fifth and final tone of the series, and write down on a response sheet whether the test tone was higher or lower than the standard. They were instructed to ignore the intervening tones.

\section{Results and Discussion}

The number of errors made by each subject in each condition was entered into a 7 (intervening tone frequency condition, within subjects) by 4 (temporal interval, between subjects) by 2 (test tone relation to last intervening tone and standard, within subjects) analysis of variance. The third analysis of variance factor 
was drawn from the joint relation between the frequencies of the standard, the last intervening tone, and the test tone. Two types of errors were defined by this relationship: "Same-direction" errors were made on trials in which the test tone frequency fell between the standard and the last interference tone; "oppositedirection" errors were made on trials in which the test tone frequency fell outside the standard and last intervening torie frequency interval. For example, an opposite-direction error occurred when the last intervening tone was of higher frequency than the standard, the test tone was of lower frequency than the standard, and the subject responded "higher." A same-direction error occurred when the last intervening tone was of higher frequency than the standard, the test tone was also higher than the standard but lower than the last intervening tone, and the subject responded "lower." The error directions were defined in a complementary fashion when the last intervening tone was lower than the standard.

All main effects and interactions were statistically significant at $\mathrm{p}<.01$ [second-order interaction, $F(18,600)=2.34, \quad M S e=6.04]$. The error rates discussed below and plotted in Figures 1 and 2 are based on the eight trials within each intervening tone condition. Over all trials the error rate was $20.6 \%$.

The intervening tone condition error rates in the same- and opposite-direction conditions are plotted in Figure 1. Newman-Keuls post hoc tests revealed that there were significantly more errors in the "low" and "high" conditions than all other conditions $(p<.01)$, but the "low" and "high" conditions were not significantly different from each other $(p>.05)$. The

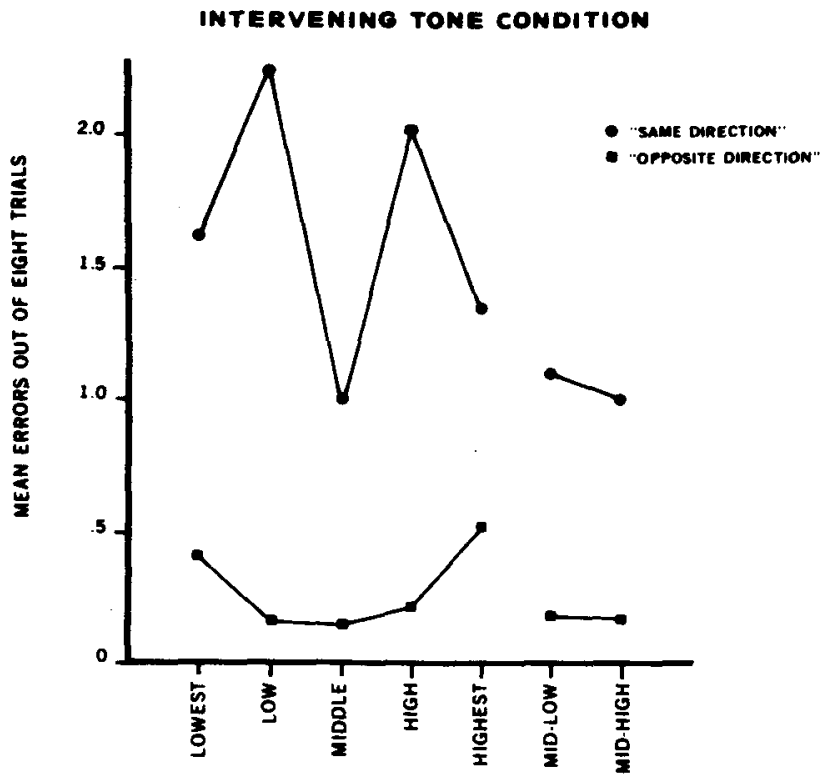

Figure 1. "Same-direction" and "opposite-direction" errors in seven intervening tone conditions. "lowest" condition had significantly more errors than the "highest" $(p<.05)$, "middle," "middle-lowest," and "middle-highest" conditions $(p<.01)$. Finally, the "highest" condition had significantly more errors than "middle," "middle-lowest," and "middle-highest" conditions $(p<.01)$.

The directional error rates seem to support an adaptation level account of the error pattern across intervening tone conditions. Subjects were much more likely to make errors on same-direction trials (2.95) than on opposite-direction trials (.54). The adaptation level model accounts for the error-direction effect by proposing that the memory of the standard was shifted toward the frequency range of the intervening tones. For example, when the intervening tones were higher than the standard and the test tone was also higher, a sufficient shift in the subject's memory of the standard toward the intervening tone frequency range would result in the subject's erroneous judgment that the test tone was lower than the standard (a same-direction error). The smallest memory shift should have occurred in the "middle" condition because the standard and intervening tone frequencies were relatively similar. The low overall error rate in the "middle" condition was consistent with this hypothesis. However, within the overall error rate, there was still a very strong difference between same-direction (.992) and opposite-direction (.152) errors.

Close examination of the "middle" tone patterns in Table 3 revealed that a memory shift toward the mean frequency of the intervening tones could account for the directional effect. Several rules guided the construction of the "middle" patterns. Each of the four standard tones was used once in the same-direction condition and once in the opposite-direction condition. (Recall that the direction conditions were defined in terms of the relation between the standard tone, the last intervening tone, and the test tone.) Within each of these conditions, two of the standards were presented with higher test tones and two with lower test tones. Intervening tones were randomly chosen from the six semitones between 622 and $830 \mathrm{~Hz}$, with the constraint that no tone could be repeated in the same pattern. As noted in the "meantest semitone difference" column of Table 3, which contains the difference in semitones between the mean of the first four tones and the test tone, this construction procedure resulted in the same-direction tone patterns with mean frequencies for the first four tones that were closer to the test tones than in the oppositedirection patterns. If the mean frequencies of the first four tones precisely represented memory for the standard, subjects should have responded correctly in both same-direction and opposite-direction conditions. However, it seemed reasonable to assume that there was some general variability in the standard memory due to other factors, and this would produce a higher error rate in the same-direction condition. 
Table 3

The "Middle" Intervening Tone Patterns (in Hertz)

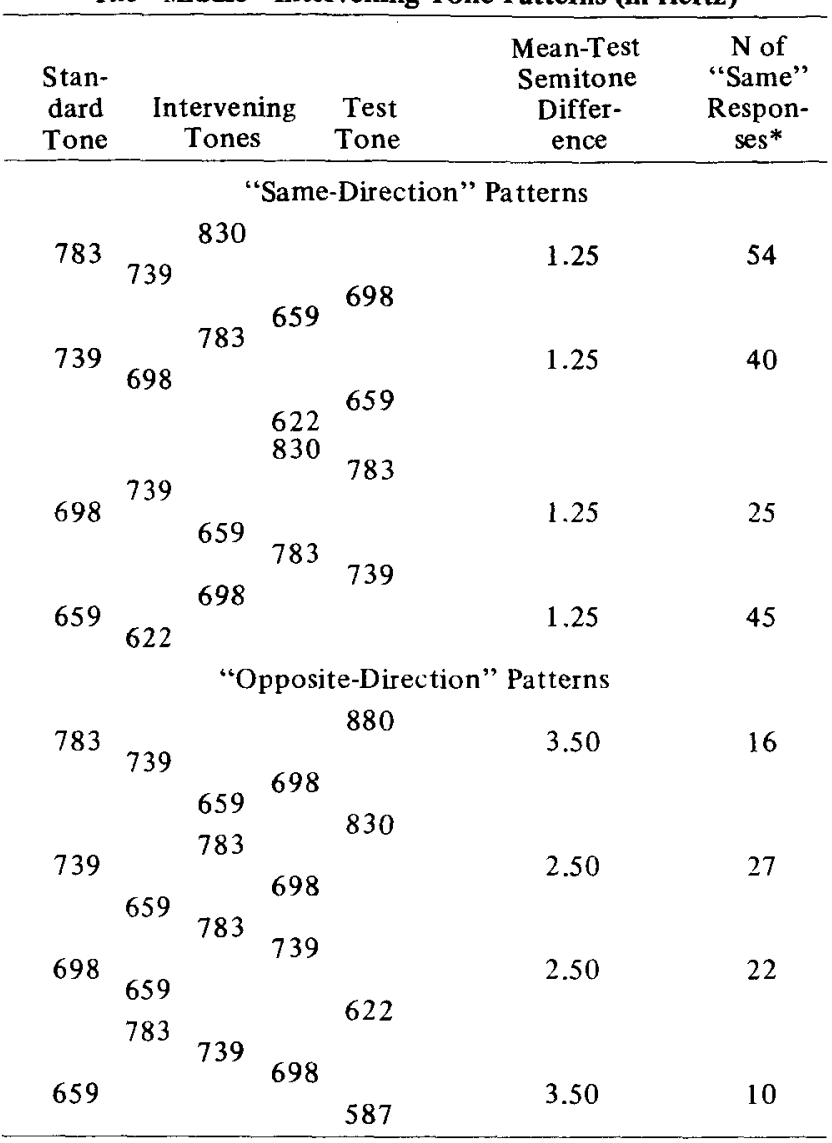

*In Experiment III

The observed decrease in same-direction error rates for the "highest" and "lowest" conditions would not be predicted on the basis of a memory shift toward the average frequency of the standard and intervening tones, since this average was considerably farther from the test tone than in the "low" and "high" conditions. However, this result was consistent with many previous adaptation level studies on other stimulus dimensions. Stimuli which were far removed from the primary test range tended to have a reduced influence on the adaptation level (Helson, 1964). In the present study, the large frequency separation of the "lowest" and "highest" intervening tones from the standard and test may have resulted in the formation of two separate "perceptual streams." Several other researchers have described the phenomenal grouping of sequentially presented tone stimuli into separate perceptual streams based on frequency range (Bregman \& Campbell, 1971; Bregman \& Rudnicky, 1975; Dowling, 1973; Van Noorden, Note 1). This hypothesis is compatible with the results in the "middle-lowest" and "middle-highest" conditions: The single extreme intervening tone may have had little additional effect on the standard memory, beyond the effects of the two "middle" intervening tones, because it was a distinct element outside the main perceptual stream.

The perceptual stream hypothesis may be extended to account for the effects of temporal interval conditions (see Figure 2). Newman-Keuls post hoc tests revealed that in overall error rates, the $300-\mathrm{msec}$ and 1 -sec intervals were not significantly diffe rent, nor were the 3 -sec and 5 -sec conditions ( $p>.05)$, but both the $3-\mathrm{sec}$ and 5 -sec conditions had significantly more errors than the 300-msec and $1-\mathrm{sec}$ conditions $(\mathrm{p}<.05)$. It is tempting to relate the greater errors in the longer intervals to previous findings of a decline in pitch recognition over longer silent retention intervals (Harris, 1952; Bachem, 1954). However, the interaction between temporal interval and intervening tone condition suggests a very different interpretation of the temporal interval main effect (see Figure 2). First, performance in the "middle" condition was not significantly different across the temporal interval groups (Newman-Keuls, $p>.05)$. Thus, the general trend of the Temporal Interval by Intervening Tone by Direction interaction may be roughly characterized as an increase in samedirection errors, except in the "middle" condition, as the temporal interval size increased. Specifically, in the
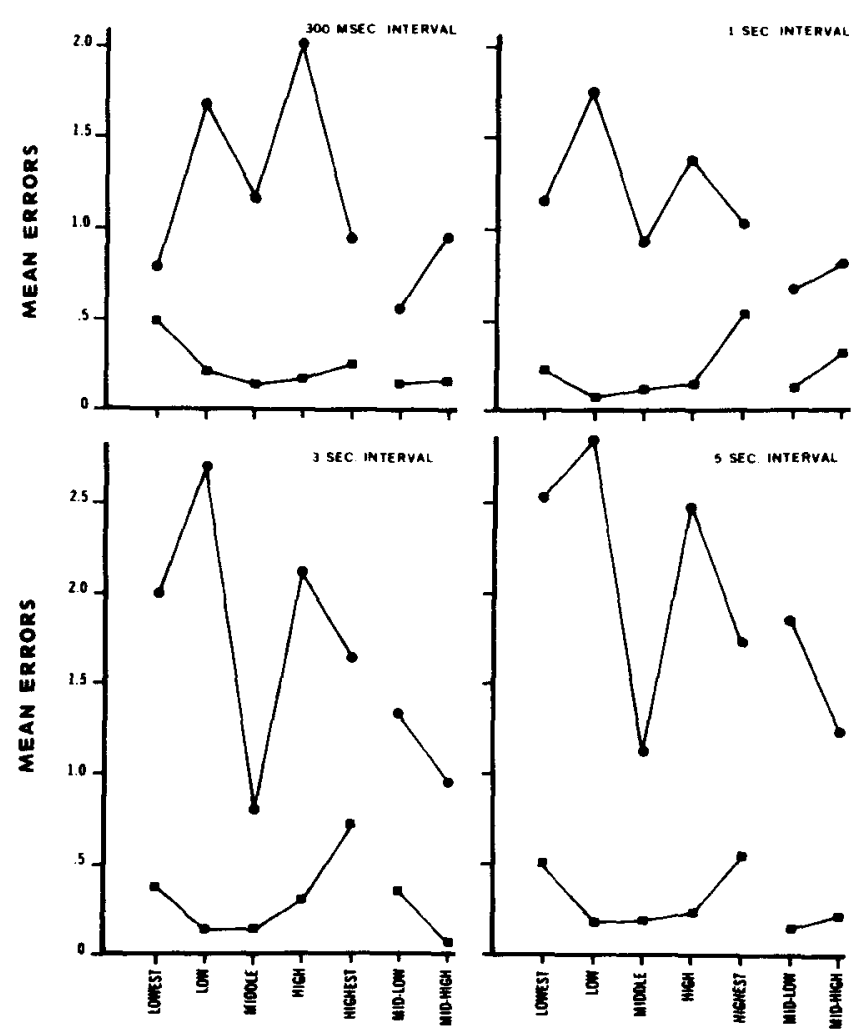

INTERVENING CONDITION

INTERVENING CONDITION

Figure 2. The interaction between direction condition, intervening tone condition, and temporal interval. 
300-msec interval group, same-direction errors were actually less frequent in the "lowest," "highest," "middle-lowest," and "middle-highest" conditions than in the "middle" condition. (This result was significant only for the "lowest" and "middle-lowest" conditions, Newman-Keuls, $p<.05$ ). In the $1-\mathrm{sec}$ group, the "middle" condition was not significantly different from the other conditions mentioned above. In the 3 and 5-sec groups, the "middle" condition error rates were lower than the other conditions.

On the basis of our own phenomenal experience and that of several others who heard all of the different temporal interval conditions, we suggest that the interaction described above resulted from a tendency for the more rapidly presented tone sequences to form more distinct frequency-based perceptual streams, in which the intervening tone stream interacted less with the standard tone/test tone stream. Further support for this hypothesis comes from a study, reported by Van Noorden (Note 1), that found an increased separation of frequency-based perceptual streams with more rapid presentations of tone sequences.

Although the memory shift and perceptual stream hypotheses provided an attractively simple account of the same-direction errors, the opposite-direction errors were not entirely consistent with the proposed standard memory shifts. A memory shift should result in a complementary relation between the numbers of opposite- and same-direction errors. Although the number of opposite-direction errors was quite small, there does not even seem to be a trend toward such a relationship (see Figure 1). Newman-Keuls tests revealed only that the "lowest" and "highest" conditions were significantly different from the others $(p<.05)$. Thus, in addition to raising a question about the memory shift hypothesis, the opposite-direction errors suggest that there may be a general non-direction-specific increase in the variability of the standard memory in the "lowest" and "highest" intervening tone conditions.

The opposite-direction error trends prompted our concern that the difference between same-direction and opposite-direction errors might have been at least partially caused by the subject's simply forgetting the standard on some trials and basing his response on the higher-lower relationship of the test tone to the last intervening tone. Some subjects expressed doubt that they had any specific memory of the standard in some of the trials. In such cases an intervening/test tone relational response strategy would inflate the samedirection error rate without reflecting an actual shift in the standard memory. Thus, for example, the "highest" and "lowest" conditions might have caused a strong nondirection-specific disruption of the standard memory, and the higher rate of same-direction errors could have been produced because the bewildered (or unattentive) subject responded with the recent and most salient higher-lower relationship between the last intervening tone and the test tone. Since we suspected that the higher-lower response measure might bias the subjects toward this type of strategy, it seemed prudent to verify the proposed shifts in standard tone memory in experiments with different response measures.

\section{EXPERIMENT 2}

The higher-lower judgment seemed to be the most likely source of a response bias in Experiment 1. Therefore, this judgment was replaced in Experiment 2 by requiring the subjects to set a continuously variable oscillator to match their memory of the standard. The method was not suitable for testing large groups of subjects so we decided to gather more extensive data from each of four subjects.

\section{Method}

Subjects. The subjects were the authors (R.O. and V.H.) and two graduate students in psychology (P.R. and A.F.) at the University of Colorado.

Stimuli and apparatus. The stimuli were the same 1-sec and 3-sec series used in Experiment 1 and two silent interval control series which included only the warning signal and the standard tones from the 1- and 3-sec tapes. The tapes were played to the subject with a SONY TC-570 tape recorder and loudspeaker. The subject made his response by setting the dial of an RCA WA 44C oscillator which was played over the same SONY TC-570 loudspeaker. The calibrated dial of the oscillator was masked with cardboard, and stops were placed on the dial to confine the settings between $440 \mathrm{~Hz}$ and $1,174 \mathrm{~Hz}$. The subject's final oscillator setting was monitored with a Hewlett-Packard electronic counter (Model 522B).

Procedure. The subjects were instructed to set the oscillator to match their memory of the standard tone. The initial oscillator dial setting was alteriated between the high and low stops on successive trials. On each trial the subject listened to the standard tone, and following either three intervening tones or a silent control interval, the experimenter turned on the oscillator at the same time the test tone would have appeared in the Experiment 1 trials. The subject then turned the oscillator dial to match his memory for the standard. He was encouraged to make a setting as rapidly as possible to minimize interference from the tones produced by the oscillator. Subjects generally made their settings in less than $5 \mathrm{sec}$. After the experimenter recorded the subject's response, the oscillator was turned off and the dial was set to the stop appropriate for the next trial.

A series of 28 practice trials from the 3-sec control tape was presented prior to the first series of experimental trials. The subjects were given feedback to correct their response if they were more than one semitone from the standard. No feedback was given in the experimental trials.

On each of 8 consecutive days the subject listened to one of the 56 trial series. The 1 - and $3-\mathrm{sec}$ tapes and their respective silent interval controls were used in the first 4 days. The silent interval control and intervening tone trial series were alternated, with two subjects beginning with the silent control trials and two beginning with the intervening tone trials. The orders of the 1and 3-sec temporal interval series were also counterbalanced over the first 4 days. The same tape orders were repeated over Days 5 though 8 , with a reversal of the high-low oscillator dial starting positions. Thus, each particular standard and intervening tone combination was tested once with the initial oscillator dial setting at the high end and once at the low end.

\section{Results and Discussion}

A 2 (temporal interval) by 7 (intervening tone) analysis of variance was performed on each subject's deviation (in cycles/second) from the standard tone 
frequency. Subject R.O. was the only one who showed a significant variation in deviations from the standard as a function of temporal interval $[1 \mathrm{sec}=8.6 \mathrm{~Hz}$, $3 \mathrm{sec}=3.9 \mathrm{~Hz} ; \quad \mathrm{F}(1,6)=5.03, \quad \mathrm{MSe}=244.6, \quad \mathrm{p}<.05]$ and intervening tone condition $[F(6,210)=2.18$, $\mathrm{MSe}=244, \mathrm{p}<.05]$. His interaction and all effects for the other three subjects were nonsignificant $(p>.05)$. The significance of the effects for R.O. was due more to his consistency than to the size of the effects. The means of the other subjects actually showed greater differences but their error terms were also much larger.

The pattern of R.O.'s mean deviation from the standard tone frequency showed a slight decrease in overestimation of the standard as the intervening tone frequencies shifted from below to above the standard. This result does not support an adaptation level model, which would predict underestimations of the standard frequency with low-frequency intervening tones and overestimations with high-frequency intervening tones. Even when R.O.'s silent control condition error of $+5.25 \mathrm{~Hz}$ is subtracted from each of the means, the trend is still in the opposite direction from the prediction of the adaptation level model. Trends in the intervening tone condition means for the other subjects also gave no indication of memory shifts consistent with the model.

The present study provided no support for the adaptation level model, but for several reasons we hesitated to consider the results of Experiment 2 as negative evidence for a memory shift in the type of tone-memory task employed in Experiment 1. Two of the subjects in the present study, V.H. and A.F., found the task very difficult, and they often expressed doubt that their responses were even approximately correct. R.O. and P.R. had more confidence in their responses, but neither subject thought the task was particularly easy. The general difficulty of the task was indicated by the subjects' standard deviations in the intervening tone and temporal interval conditions. Several points may be made from these measures of variance. First, there were considerable individual differences in the overall magnitude of the standard deviations. Subject V.H.'s standard deviations averaged over three times larger than those of R.O., and this difference did not seem to be due to attentional or motivational factors. Second, except for A.F., the standard deviations in the 1 -sec condition were consistently smaller than in the 3-sec condition. Third, there was no consistency over subjects in the pattern of standard deviations across the different intervening tone conditions.

The magnitude of the silent control standard deviations suggested a possible reason for the lack of consistent patterns in the mean frequency responses and standard deviations for the different intervening tone conditions. The silent interval control standard deviations were not very much smaller than the mean of the intervening tone condition standard deviations. The subjects reported that considerable interference with the standard occurred from the shifting tones as they made their response with the oscillator. The interference may have reduced the subjects' performance to a level where the intervening tone condition effects were obscured. Therefore, we designed the third experiment to avoid both the potential intervening/test tone relational response biases in Experiment 1 and the excessive memory disruption from the response measure in Experiment 2.

\section{EXPERIMENT 3}

Since it was suspected that the higher-lower judgment might be producing a response bias in Experiment 1, in Experiment 3 the subjects were told to respond "same" or "different." The same 3-sec interval tapes were used as in Experiment 1, so there were no actual "same" pairs of standard and test tones. However, an increase in "same" responses was expected for conditions in which the standard memory was generally disrupted, and if the disruption was direction specific, there should be more same-direction "same" responses than opposite-direction "same" responses.

The results of Experiment 2 made us acutely aware of the magnitude of individual differences in tone-memory tasks. Therefore, data was obtained on the subjects' musical background and their self-evaluation of their musical skills. This variable was then included in the analyses of the subjects' responses.

\section{Method}

Subjects. One hundred and twenty introductory psychology students at the University of Colorado participated in the experiment in partial fulfillment of a course research participation requirement.

Stimuli and apparatus. Both orders of the 3-sec interval tapes employed in Experiment 1 were used in the present experiment. They were played to the subjects with a SONY TC-570 tape recorder and loudspeaker.

Procedure. Twelve groups of 10 subjects listened to the 3 -sec interval tapes. They were seated about $10 \mathrm{ft}$ from the loudspeaker and were told to listen carefully to the standard tone, ignore the intervening tones, compare the final test tone with their memory for the standard, and write "same" or "different" on the response sheet. They were told that there would be "some" standard-test pairs in the trial series that would be the same and "some" that would be different in frequency. At the end of the trial series, the subjects were asked to describe their musical experience in some detail (years of training, etc.) and to give a self-rating of their current musical competence on a scale of very poor, poor, fair, good, and very good.

\section{Results and Discussion}

The 120 subjects' responses to the musical experience and competence questions were scaled in the following manner. One point was given for each year of musical training or active involvement in music. The ratings of competence were assigned from 1 (very poor) to 5 (very good) points. The point totals for training and compe- 
tence provided the basis for dividing the subjects into "musical" and "nonmusical" groups of 60 subjects. The mean point scores for the musical and nonmusical groups were 9.70 and 2.03 , respectively, and the point score boundary between the groups was 5.5 .

The subjects' responses were entered into a 2 (musical experience, between subjects) by 2 (direction of test tone, within subjects) by 7 (intervening tone condition, within subjects) analysis of variance. Musical and nonmusical subjects, respectively, responded "same" 1.22 and 1.62 times out of eight trials $[F(1,116)=15.46$, $\mathrm{MSe}=4.19, \mathrm{p}<.01]$. A "same" response can be considered an error since all standard and test tone pairs were separated by two semitones. This suggests that the musical subjects' tone memory was more accurate, although differences in criteria could have influenced the number of "same" responses in the two groups.

Aside from their overall differences in accuracy, the musical and nonmusical subjects were not significantly different in interaction with the intervening tone conditions $[F(6,696)=1.19, \mathrm{MSe}=.979, \mathrm{p}>.05]$ or directional effects $[\mathrm{F}(1,116)=.406, \mathrm{MSe}=1.14, \mathrm{p}>.05]$. Musical subjects seemed to show a slightly more consistent separation between same-direction and opposite-direction errors over the intervening tone conditions, but, in general, the performance of musical and nonmusical subject groups was qualitatively similar.

"Same" responses occurred significantly more often when the test tone was between the frequencies of the standard and last intervening tone [same-direction condition $=1.59$, opposite-direction condition $=1.24$; $\mathrm{F}(1,116)=44.80, \quad \mathrm{MSe}=1.14, \mathrm{p}<.01]$. This result seemed to provide support for the memory shift prediction of the adaptation level model in a task which was not subject to the potential relational response biases in Experiment 1. However, the direction condition and apparent memory shift interacted with intervening tone condition $[F(6,696)=7.68, \mathrm{MSe}=.865, \mathrm{p}<.01]$ in a way that seemed inconsistent with the adaptation level model. The pattern of this interaction may be observed in Figure 3. Much of the variance in the interaction is associated with the relatively large difference between same-direction and opposite-direction error rates in the "middle" condition (Newman-Keuls, $p<.05$ ), when it had been expected from Experiment 1 that the directional effect would be stronger in the "low" and "high" conditions. Following the argument presented in Experiment 1, if the averaged frequency of the first four tones tended to represent the subjects' memory of the standard in the "middle" condition (see Table 3), they would be much more likely to respond "same" in the same-direction trials. This explanation was consistent with the results in the "middle"condition, but it was less clear how this hypothesis could account for the subjects' performance in the other intervening tone conditions. There was a significant directional effect for the "middle-lowest" and "middle-highest" intervening tones over all subjects $($ Newman-Keuls. $p<.05)$,

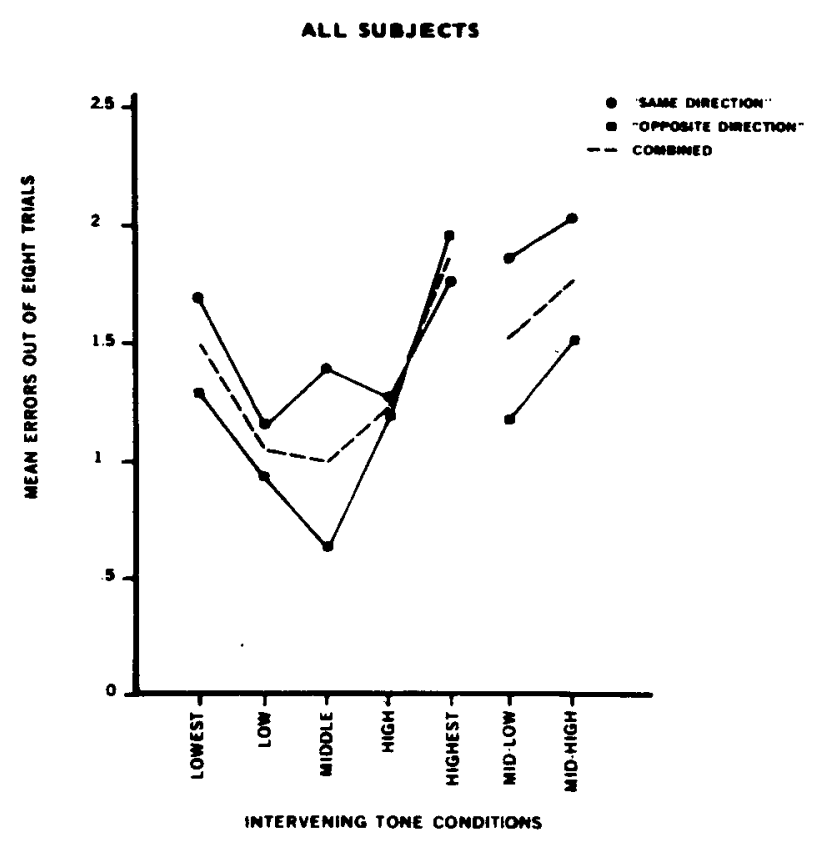

Figure 3. Mean number of "same" responses for all subjects in the intervening tone conditions.

and the musical subjects had a significant directional effect in the "low" condition (Newman-Keuls, $p<.05$ ). However, there was no significant difference in directional errors in the "lowest," "low," "high," and "highest" conditions for the nonmusical subjects or in the "lowest," "high," "highest," and "middle-highest" conditions for the musical subjects. It might be argued that the directional effects were obscured in these conditions because the standard tone memory was pulled so far away from the test tone that it was very dissimilar from both the same-direction test tone and the opposite-direction test tone. This argument has the obvious flaw that such a large shift in the standard memory would result in a reduced incidence of "same" responses as the standard memory was pulled away from the test tone frequency, while in fact the same responses were more frequent in the extreme intervening tone conditions.

"Same" responses (represented by the dashed curve in Figure 3) were not significantly different in the "low," "middle," and "high" conditions (NewmanKeuls, $p>.05$ ), and all three conditions resulted in significantly fewer "same" responses than the "lowest," "highest," "middle-lowest," and "middle-highest" conditions (Newman-Keuls, $p<.05)$. Since it was argued above that a strong directional memory shift would produce a lower rate of "same" responses in the extreme intervening tone conditions, some other factor must be responsible for the observed higher rate.

Some subjects reported using a relational encoding system when they were questioned about their strategies in the task. They tried to remember the up-down 
sequence and separation of the tones and they felt that this was easier with the "middle" intervening tones than the more extreme conditions. Thus, instead of ignoring the intervening tones as instructed, some subjects may have used them as a structural support for the standard tone memory. Other subjects said they did not consciously use this type of strategy. In either case, the subjects' introspective reports should be considered as only suggestive evidence for the relational model.

The relational model's application to the present results is consistent with previous data on the reproduction of transposed intervals between two tones (Attneave \& Olson, 1971). Subjects in this study listened to tone patterns which were alternations of two different tones and they were required to transpose these patterns to another frequency range. For subjects who did not have a precise musical interval coding system, the variability in the reproduced interval size was proportional to the size of the interval. In the present tone-memory study, subjects may have encoded the intervals between the standard and intervening tones rather than or in addition to their absolute pitch. Thus, the larger intervals in the "highest" and "lowest" conditions would be less precisely defined in memory and result in a higher error rate.

We have failed to arrive at a satisfying explanation for the relatively high incidence of "same" responses and strong directional effects in the "middle-lowest" and "middle-highest" conditions. In contrast to the "middle" condition, which contained pattern differences that could account for the directional effects, an examination of the mean frequencies of the standard and first two intervening tones in the "middlelowest" and "middle-highest" conditions showed no difference in their mean distance from the test tone for the same-direction and opposite-direction trials. Thus, the directional effect must be based on the single "highest" or "lowest" intervening tone, and it seems puzzling that the directional effect for a single extreme tone would be stronger than the effect for three extreme intervening tones. An additional problem is raised by the high overall "same" response rate. The relational coding hypothesis described earlier would seem to predict a better memory for the standard and fewer "same" responses when there were two "middle". range intervening tones to provide support for the standard. However, performance in the "middle-lowest" and "middle-highest" conditions was, respectively, quite close to the "lowest" and "highest" conditions (see the dashed curve in Figure 3).

Before an attempt is made to integrate the results of our three studies, there is a fourth experiment that should be considered. While our Experiment 3 was in progress, a "same-different" study by Deutsch (1974) on frequency range interference effects came to our attention. Both the design and results were different from ours in several respects. Deutsch's temporal interval condition was the same as our $300-\mathrm{msec}$ condition in Experiment 1, but there were eight intervening tones; the standard and test were identical on half the trials and differed by only one semitone in the other half. Frequencies of the intervening tones ranged from 129 to $977 \mathrm{~Hz}$, with the standard and test tones between 274 and $517 \mathrm{~Hz}$. The four intervening tone conditions included tones drawn from the same octave as the standard and test, one octave above, one octave below, and one condition with intervening tones both an octave above and an octave below the standard. Each of these conditions was used separately in 12 trial blocks. The percentages of errors in the conditions were: same octave $=33.5$, high octave $=28.1$, low octave $=25.6$, and high-low octaves $=38.3$. The most striking difference between Deutsch's study and ours was her relatively high error rate results when the intervening tones were drawn from the range closest to the standard and test. Unfortunately, the experimental methods and stimuli differed in so many ways that it was impossible to determine the basis for our differences in overall error rate patterns, and the subjects' responses in Deutsch's experiment were not analyzed for directional effects.

The variation in results over our three experiments and Deutsch's (1974) suggests that there are several important stimulus and task variables that influence tone memory. Given these differences in results, what generalizations can be drawn from our tone memory studies?

\section{GENERAL DISCUSSION}

The conclusinn to Deutsch's (1974, p. 223) frequency range interference study noted that "interference in memory for the pitch of a tone may be due to a variety of factors." One set of factors is related to the fact that tone sequences tend to be structured and remembered as patterns within a muscial coding system (Attneave \& Olson, 1971; Deutsch, 1969). Another set of factors involves the combination of adaptation level effects, perceptual stream formation, and relational encoding strategies in the present studies. The construction of a general model for tone memory is further complicated by individual differences and the unique effects of experimental task demands. The results of the present experiments reflect these complications, and the varied methods provide further insights into some of the important factors in tone memory. The following discussion begins by examining the consistent implications in the results of Experiments 1 and 3 for the adaptation level and relational encoding hypotheses. Attention is then directed to apparent inconsistencies in the results over all three experiments.

Evidence for a shift in the standard tone memory toward the intervening tones was present in Experiments 1 and 3. Although the precise magnitude of this shift could not be determined, it was shown to vary as a function of the presentation rate and the frequency 
distance of the intervening tones from the standard and test tones. Both these variables seemed to contribute to the separation of intervening tones and standardtest pairs into separate perceptual streams, with greater frequency distances and more rapid presentations tending to reduce the amount of interference and increase the phenomenal distinction between the "streams." In addition to the directional shifts in memory, there was evidence for a general increase in variability and decrease in the strength of the standard tone memory in the "highest" and "lowest" conditions. This effect was indicated in Experiment 1 by the higher incidence of opposite-direction errors and in Experiment 3 by an increased number of "same" responses in these intervening tone conditions. The most reasonable hypothesis for this effect seems to be the failure of the more distant intervening tones as a relational reference for the standard memory.

In general, the interaction of the directional memory shifts and memory variability differences over the intervening tone conditions is characterized by a strong directional effect but relatively low variability for the standard memory in the "middle" condition, and a weaker directional effect but higher variability for the standard memory in the "highest" and "lowest" conditions. Specification of these effects in the other intervening tone conditions depends on the experimental task. Strong directional effects were noted in all Experiment 1 intervening tone conditions, while in Experiment 3 the effects were strong only in the "middle," "middle-lowest," and "middle-highest" conditions. Since the stimuli were identical in the two experiments, the shift in the subjects' judgment from "higherlower" to "same-different" must have accounted for the change in performance. We could not determine precisely how these differences in judgment gave different results. They may have initiated different strategies for coding the standard and intervening tones, or the differences could be due to response strategies such as a reliance on the higher-lower relationship of the last intervening tone and the test tone.

$A$ second difference between the results of Experiments 1 and 3 was in the overall error rates in the "high" and "low" conditions. In Experiment 1, the largest error rates were in these conditions, resulting in an $M$-shaped curve of interference effects. In Experiment 3, the "high" and "low" errors were slightly and nonsignificantly more frequent than "middle" errors, but the "lowest" and "highest" errors were much more frequent, thus producing a U-shaped function. Either the encoding or the response strategy differences referred to above may account for this difference between experiments.
The results of Experiment 2 do not fit any of the models we have proposed to account for the effects in the other studies. It was noted that the silent control performance was almost as variable as performance with the intervening tones, and that the tones produced by the oscillator may have obliterated any additional interference effects. However, it might also be argued that the task demands of tone matching and production led to different encoding or retrieval operations which enabled the subjects to ignore the intervening tones. While this issue cannot be resolved here, it was raised to further emphasize the importance of task variables in tone-memory studies.

The different methods of Experiments 1, 2, and 3 have produced a wide variety of results. At this point, we have been able to account for many of the results with the adaptation level and relational encoding models. Further research is needed to specify the reasons for judgment task effects and to more precisely determine the magnitude of standard tone-memory shifts.

\section{REFERENCE NOTE}

1. Van Noorden, L. P. A. S. Rhythmic fission as a function of tone rate. IPO Annual Progress Report (No. 6). Eindhoven. Netherlands: Institute for Perception Research, 1971. Pp. 9-12.

\section{REFERENCES}

Attneave, F., \& Olson, R. K. Pitch as a medium: A new approach to psychophysical scaling. American Journal of Psychology, 1971, 84, 147-165.

BACHEM, A. Time factors in relative and absolute pitch determination. Journal of the Acoustical Society of America. 1954, 26, 751-753.

Bregman, A. S., \& Campbell, J. Primary auditory stream segregation and perception of order in rapid sequence of tones. Joumal of Experimental Psychology, 1971, 89, 244-249.

Bregman, A. S., \& Rudnicky, A. I. Auditory segregation: Stream or streams? Journal of Experimental Psychology: Human Perception and Performance, 1975, 1, 263-267.

Deutsch, D. Music recognition. Psychological Review, 1969. 76. $300-307$.

Deutsch. D. Generality of interference by tonal stimuli in recognition memory for pitch. Quarterly Joumal of Experimental Psychology, 1974, 26, 229-234.

Deursch, D. Auditory memory. Canadian Journal of Psychology, 1975, 29, 87-105.

DEUTSCH, D., \& FEROE, J. Disinhibition in pitch memory. Perception \& Psychophysics, 1975, 17, 320-324.

Dowling, W. J. The perception of interleaved melodies. Cognitive Psychology, 1973, 5, 322-337.

Harris, J. D. The decline of pitch discrimination with time. Journal of Experimental Psychology, 1952, 43. 96-99.

Helson, H. Adaptation-level theony. New York: Harper \& Row, 1964.

(Received for publication February 9, 1976; revision received June $9,1976$. ) 